\title{
Persistence of Symptoms in Primary Somatoform Vertigo and Dizziness
}

\author{
A Disorder "Lost" in Health Care? \\ Regine Tschan, PhD, * Christoph Best, MD, $\dagger$ Jörg Wiltink, MD, * Manfred E. Beutel, MD, *
Marianne Dieterich, MD, $\neq$ and Annegret Eckhardt-Henn, MD
}

\begin{abstract}
The aim of this study was to perform a 3-year follow-up of primary somatoform vertigo and dizziness (SVD) regarding health care use and treatment. Ninety-two patients with dizziness underwent detailed vestibular neurophysiological testing and a Structured Clinical Interview for Diagnostic and Statistical Manual of Mental Disorders, Fourth Edition. Psychometric assessments comprised the Vertigo Symptom Scale, the Vertigo Handicap Questionnaire, the SCL-90-R, and the Short-Form-36 Health Survey. At the 3-year follow-up, 65 patients with primary SVD (anxiety, $n=29$; depression, $n=14$; somatoform disorders, $n=22$ ) were reassessed ( $70.7 \%$ response). The patients improved in symptom severity $(p<0.05)$, handicap $(p<0.01)$, and physical quality of life (QoL; $p<0.05)$ but showed no change in emotional distress. A total of $63.1 \%$ (of $n=65$ ) had ongoing SVD. A total of $69.2 \%$ (of $n=65$ ) received different forms of treatments. A total of $46.1 \%$ (of $n=65$ ) searched redundant medical diagnostic procedures. The patients with decreased coping capacity over time obtained the best prognosis. Primary SVD is an ineffectively treated disorder. Recommendations for specific complaint-oriented psychotherapy programs were given.
\end{abstract}

Key Words: Vertigo, dizziness, psychotherapy, handicap, quality of life.

(J Nerv Ment Dis 2013;201: 328-333)

$P$ rimary somatoform vertigo and dizziness (SVD) as a correlate of a psychopathological process is a common diagnosis in psychosomatic health care (Kroenke et al., 2000; Staab and Ruckenstein, 2007b). Primary SVD is defined as the superordinate classification of different mental subgroups (anxiety, depressive, or somatoform disorders). Diagnostically, no vestibular deficit or dysfunction in medical history could be found. Trigger factors for the onset of SVD can result from critical life events, emotional distress, or relational or psychodynamic conflicts (Eckhardt-Henn and Dieterich, 2005; Yardley, 2000).

In contrast to primary SVD, secondary SVD is defined as a mental comorbidity after an initial (and meanwhile centrally compensated or peripheral remitted) vestibular disorder resulting from individual maladaptive coping (Best et al., 2009). For example, phobic postural vertigo as a subtype of secondary SVD can be a result of

\footnotetext{
* Clinic of Psychosomatic Medicine and Psychotherapy, University Medical Center of the Johannes Gutenberg-University Mainz, Mainz, Germany; †Department of Neurology, University Medical Center of the Johannes Gutenberg-University Mainz, Mainz, Germany; †Department of Neurology, Ludwig-MaximiliansUniversity, Munich, Germany; and §Department of Psychosomatic Medicine, Bürgerhospital, Stuttgart, Germany.

Send reprint requests to Regine Tschan, $\mathrm{PhD}$, Clinic of Psychosomatic Medicine and Psychotherapy, University Medical Center of the Johannes Gutenberg-University Mainz, Untere Zahlbacher Str. 8, 55131 Mainz, Germany. E-mail: regine.tschan@unimedizin-mainz.de. Copyright (C) 2013 by Lippincott Williams \& Wilkins

ISSN: 0022-3018/13/20104-0328

DOI: $10.1097 / \mathrm{NMD} .0 \mathrm{~b} 013 \mathrm{e} 318288 \mathrm{e} 2 \mathrm{ad}$
}

catastrophizing beliefs and anxious avoidance during states of vertigo attacks (Huppert et al., 2005; Kapfhammer et al., 1997).

Epidemiologically, more than $30 \%$ of all patients with vertigo or dizziness have primary SVD (Kroenke et al., 2000). Thereof, $45 \%$ showed comorbid anxiety; $30 \%$, depressive disorder; and $25 \%$, somatoform disorders (Eckhardt-Henn et al., 2003). Recent studies explored specific epidemiological data for vertigo or dizziness in community-based and primary care-based settings. Prevalence rates range from $20 \%$ up to $30 \%$ of affected people in the general population (Nazareth et al., 2006; Neuhauser et al., 2008; Wiltink et al., 2009). Up to $80 \%$ of vertigo or dizziness resulted in medical consultations, restrictions of daily activities, or sick leaves (Nazareth et al., 2006; Neuhauser et al., 2005). In a UK sample of working-aged people with dizziness, $40 \%$ seek help through their general practitioners, but only one third received some form of treatment (Yardley et al., 1998). Anxiety-induced SVD revealed higher health care use than did vertigo or dizziness without comorbid anxiety or phobic disorders (Wiltink et al., 2009). Posing a risk for a chronic course, primary SVD leads to extensive handicaps and restrictions in quality of life (QoL; Kroenke et al., 2000; Staab and Ruckenstein, 2007b). Because no dizzinessspecific psychotherapy programs are available, little is known about health care use and psychotherapeutic outcome. The purpose of this prospective study was to perform a 3-year follow-up of patients with primary SVD regarding persistence of symptoms, handicap, and coping. Clinical characteristics, health care use, treatment, and their implications for psychotherapy programs were analyzed.

\section{PATIENTS AND METHODS}

In an interdisciplinary long-term study, we examined a total of 92 consecutive patients with primary SVD during a course of 3 years. At baseline measurements (T0), 39 patients $(42.4 \%)$ were diagnosed with an anxiety disorder (ICD-10: F40, F41); 18 patients (19.6\%), with a depressive disorder (ICD-10: F32, F33, F34.1); and 35 patients $(38 \%)$, with a somatoform disorder (ICD-10: F45). The total sample included 44 women and 48 men; the mean (SD) age was 41.8 (13.3) years. The exclusion criteria were regular intake of any medication affecting the CNS, an acute disease of the CNS, an acute psychotic disorder, current psychotherapy, and inadequate knowledge of the German language. This study was approved by the local ethics committee, according to the criteria of the Declaration of Helsinki (1964). The patients were included in this study after they had given their written informed consent.

All participants underwent detailed diagnostic procedures (T0) consisting of a clinical neurological examination; a neuro-orthoptic analysis; and neurophysiological vestibular laboratory testing including an electro-oculography with caloric irrigation (Honrubia, 1994), measurements of the subjective visual vertical, and determination of ocular torsion by fundus photographs (Dieterich and Brandt, 1993). The psychosomatic examination comprised a psychometric test battery measuring dizziness-related somatic and mental symptoms. Assignment to the different subgroups of SVD was performed using the 
Structured Clinical Interview for Diagnostic and Statistical Manual of Mental Disorders, Fourth Edition (DSM-IV), Axis I disorders (Wittchen et al., 1989). After the diagnostic examinations, all patients with SVD received short psychoeducation and a specific recommendation for psychotherapy. Table 1 gives an overview about the psychotherapeutic interventions.

\section{Survey Questionnaires}

The participants were asked to complete a survey questionnaire including sociodemographic data, health care use, and subjective health outcome. Health care use was assessed by the "number of medical disciplines for further diagnostic procedures" and by the "number and forms of treatment." Next to the Vertigo Symptom Scale (VSS), persistence of SVD was assessed using the question "Do you currently suffer from ongoing vertigo or dizziness?" The answer format was a 3-point Likert scale ranging from 0 , "no dizziness complaints"; 1, "rare dizziness complaints"; to 2, "frequent dizziness complaints."

\section{Vertigo Symptom Scale}

Vertigo- or dizziness-related symptoms were examined using the German version of the VSS. The questionnaire consists of 34 items measuring the frequency and the quality of dizziness (vertigo, imbalance, and diffuse dizziness) and its concomitant symptoms. Two main subscales can be derived: vertigo and related symptoms (VER) examines the symptom severity, whereas somatic anxiety and autonomic arousal (AA) assesses the accompanying anxiety-related symptoms. As unit of measurement, the sum scores of the two scales are calculated. The VSS-VER and the VSS-AA have good internal consistencies (Cronbach's $\alpha$ : VER, 0.79; AA, 0.89; Tschan et al., 2008).

\section{Vertigo Handicap Questionnaire}

The Vertigo Handicap Questionnaire (VHQ) measures the handicapping consequences of vertigo and dizziness, including disease-specific limitations on physical activities and their impact on social life and leisure time activities. Some of the items assess social support, stigmatization, and distress caused by dizziness. It therefore reflects the restrictions patients experience in their daily life activities caused by inadequate coping with vertigo or dizziness. The total sum

TABLE 1. Table of Contents for Psychotherapeutic Short-term Intervention

Sessions 1-2 Diagnostic procedures and feedback

a) Initial clinical interview for the diagnosis of SVD

b) Structured Clinical Interview for DSM-IV (SCID-I) to diagnose the specific subgroup of SVD

c) Feedback about the clinical outcome

Session 3 Disease models and mechanism

a) Psychoeducation about the causes and the maintaining mechanisms of SVD

b) Psychoeducation about the significant role of stress, anxiety, and avoidance behavior relating to ongoing dizziness and decreased QoL

Session 4 Treatment recommendations and their mechanisms of action

Obligatory: specific recommendations for psychotherapy

a) Cognitive-behavioral or psychodynamic psychotherapy

b) Outpatient or inpatient psychotherapy

Optionally: recommendation for psychopharmacological treatment (e.g., SSRI), relaxation, or vestibular rehabilitation

SSRI indicates selective serotonin reuptake inhibitor score ranges from 0 to 100 points (percentage of handicap). The VHQ has a good internal consistency (Cronbach's $\alpha=0.92$; Tschan et al., 2010).

\section{The SCL-90-R}

The German version of the SCL-90R is a standardized selfreport instrument measuring psychopathology on nine scales: somatization, obsessive-compulsive, interpersonal sensitivity, depression, anxiety, anger-hostility, phobic anxiety, paranoid ideation, and psychoticism. The Global Severity Index (GSI) indicates the dimension of emotional distress. The subscales have good internal consistencies (Cronbach's $\alpha=0.75-0.97$; Franke, 1995).

\section{Short-Form-36 Health Survey}

Subjective QoL was assessed using the German version of the Short-Form-36 Health Survey (SF-36) as an instrument for evaluating various aspects of health status. It examines two main components by eight health-related concepts: The physical health component (PHC) consists of the subscales physical functioning, role-physical, bodily pain, and general health; the mental health component (MHC) contains the subscales mental health, role-emotional, social functioning, and vitality. As unit of measurement, the total sum for both scales is calculated. The subscales have satisfactory to good internal consistencies (Cronbach's $\alpha=0.57-0.97$; Bullinger and Kirchberger, 1998).

\section{Statistical Analysis}

Statistical analyses were performed by SPSS (Statistical Package for the Social Sciences) for Windows (version 15.0) by nonparametric (Fisher's exact test and chi-square test) and parametric procedures ( $t$-test, analysis of variance [ANOVA]). For post hoc comparisons, we used the Scheffe's procedure. We defined the level of significance at $p<0.05$; we additionally report the results with higher levels of confidence $(p<0.01, p<0.001)$. Because of the exploratory character of this study, we did not perform alpha adjustment.

\section{RESULTS}

\section{Dropout Analysis}

From baseline to the 3 -year follow-up, we could reassess a total of 65 patients. A total of $29 \%(n=27)$ dropped out because of the following reasons: no valid address $(n=9)$ and not interested in further examination $(n=18)$. The participants who dropped out were slightly younger than the participants from the study sample (42.2 vs. 43.7 years; $p<0.05$ ). Table 2 shows the sociodemographic data of the sample.

With regard to the psychometric data, the participants who dropped out $(n=27)$ reported higher levels of global distress (GSI: $\mathrm{T}[90]=2.34 ; p=0.021)$ than did the study sample $(n=65)$. Moreover, the participants who dropped out had higher scores on the VER subscale of the VSS (VSS-VER: T[89] $=2.34 ; p=0.021$ ). No significant differences between the study and the dropout sample could be obtained regarding QoL (SF-36), dizziness-specific handicap (VHQ), and the AA subscale of the VSS (VSS-AA).

\section{Distress, QoL, Vertigo, or Dizziness Symptoms and Handicap Through the 3-Year Follow-up Period}

After the 3-year follow-up period, 24 study participants (36.9\% of $n=65$ at T1) did not or only seldomly experienced dizziness. Overall, the patients judged their physical health as improved $(p=0.01)$, but not their mental health. Global distress (GSI) did not change during the 3 years. The patients reported that their dizzinessspecific handicap improved $(p=0.001)$ and that they experienced fewer vertigo or dizziness-related symptoms $(p<0.05)$. Somatic AA 
TABLE 2. Patient Characteristics: Sociodemographic Data

\begin{tabular}{|c|c|c|c|c|}
\hline & Study Sample $(n=65)$ & Dropout Sample $(n=27)$ & Total $(N=92)$ & \\
\hline Sex (female) & $30(46.2 \%)$ & $14(51.9 \%)$ & $44(47.8 \%)$ & Fisher's exact test, $p=0.653$ \\
\hline Age, mean (SD), yrs & $47.78(11.88)$ & $41.50(11.24)$ & $45.94(11.98)$ & $\mathrm{T}(90)=2.34, p=\mathbf{0 . 0 2 1}$ \\
\hline Partnership & $52(80.0 \%)$ & $20(74.1 \%)$ & $72(76.3 \%)$ & Fisher's exact test, $p=0.583$ \\
\hline Education & & & & $\chi_{3}^{2}=0.49, p=0.785$ \\
\hline Lower than 10th grade & $24(36.9 \%)$ & $10(37.0 \%)$ & $34(37.0 \%)$ & \\
\hline Completed 10th grade & $22(32.3 \%)$ & $7(26.0 \%)$ & $28(30.4 \%)$ & \\
\hline Completed high school & $20(30.8 \%)$ & $10(37.0 \%)$ & $30(32.6 \%)$ & \\
\hline Pension $^{\mathrm{a}}$ & $7(10.9 \%)$ & $3(11.1 \%)$ & $10(11.0 \%)$ & Fisher's exact test, $p=1.000$ \\
\hline Unemployed $^{\mathrm{a}}$ & $6(9.4 \%)$ & $3(11.1 \%)$ & $9(9.9 \%)$ & Fisher's exact test, $p=1.000$ \\
\hline Diagnosis & & & & $\chi_{2}^{2}=0.88, p=0.645$ \\
\hline Anxiety disorder & $28(40.7 \%)$ & $11(43.1 \%)$ & $39(42.4 \%)$ & \\
\hline Depression & $14(21.5 \%)$ & $4(14.8 \%)$ & $18(19.6 \%)$ & \\
\hline Somatoform disorder & $23(35.4 \%)$ & $12(44.4 \%)$ & $35(38.0 \%)$ & \\
\hline
\end{tabular}

Values are presented as number (percentage) unless otherwise indicated.

${ }^{\mathrm{a}}$ Missing data, $n=1$.

did not change during the follow-up period. Pre-post effect sizes for significant results were only small. Table 3 gives an overview.

\section{Health Care Use Through the 3-Year Follow-up Period}

Although all patients underwent a detailed neurological and psychosomatic examination, 30 patients $(46.2 \%$ of $n=65$ to T1) received further clinical examination in different fields: neurological $(40 \%)$, otolaryngological $(31.3 \%)$, orthopedic $(28.1 \%)$, internal medicine $(17.2 \%)$, ophthalmic $(18.8 \%)$, psychosomatic $(24.6 \%)$, and others (4.6\%). Additional 45 participants $(69.2 \%$ of $n=65)$ reported to have been treated for dizziness during the 3 -year follow-up period by different methods (physiotherapy, relaxation, [psycho]pharmacological treatment, and psychological interventions). Although all patients received the recommendation to undergo psychotherapy because of primary SVD, $36(62.5 \%$ of $n=65)$ received outpatient psychotherapy (one third $<12$ sessions) and a proportion of $16(28.6 \%$ of $n=65)$ received inpatient psychotherapy ( $>3$ weeks).

\section{Changes in Distress, QoL, and Vertigo Symptoms During the 3-Year Follow-up Depending on Changes in Vertigo- or Dizziness-Specific Handicap}

To compare the successful patients regarding vertigo- or dizziness-specific handicap in the long run (baseline to the 3-year follow-up) with those without success, we divided the study sample into three groups by the following definition: increase, the patients with a worsening of handicap on the VHQ scale $(n=20)$; unchanged, the patients with no change and a decrease of 15 points $(n=21)$; decrease, loss of 15 points and more $(n=20)$. Four patients did not provide valid data on the VHQ. Therefore, they were omitted for the following analyses. Figures 1 to 3 show changes in distress, QoL, and vertigo or dizziness symptoms during the 3 -year follow-up depending on changes in handicap.

Dependent on the changes in vertigo- or dizziness-specific handicap (VHQ) during the 3-year follow-up, the patients with increased handicap also increased in distress (GSI) and differed significantly from the patients with decreased handicap (GSI; $p<0.001$ ). The patients with increased handicap had increased vertigo or dizziness symptoms (VSS-VER). They significantly differed from the patients with unchanged or decreased handicap (VSS-VER; $p<0.05$ ). The patients with increased handicap also increased for autonomic anxiety (VSS-AA) and differed significantly from the patients with decreased handicap (VSS-AA; $p<0.05$ ). The patients with decreased handicap 3 years after admission improved in physical (PHC; $p<0.001$ ) and mental QoL (MHC; $p<0.01$ ). For the PHC, they significantly differed from the patients with increased handicap, and, in addition, for mental QoL, they significantly differed from those patients with unchanged VHQ during the 3-year follow-up.

\section{DISCUSSION}

We initially explored 92 patients from our interdisciplinary outpatient unit for primary SVD. Illness onset was within the last 6 months. The patients received detailed neurological and psychosomatic diagnostic examinations, diagnostic explanation, short psychoeducation, and a specific recommendation for outpatient or inpatient psychotherapy. Three years after admission, we reassessed 65 patients by postal psychometric survey. We were interested in both persistence of symptoms, for example, symptom severity, mental comorbidity,

TABLE 3. Distress, QoL, Vertigo, or Dizziness Symptoms and Handicap Through the 3-Year Follow-up Period ( $n=59-63)$

\begin{tabular}{lcccc}
\hline & Intake & 3-Year Follow-up & Significance & ES (Pre-Post) \\
\hline GSI (SCL-90-R) & $0.71(0.46)$ & $0.63(0.44)$ & $\mathrm{T}(63)=1.21, p=0.230$ & 0.137 \\
PHC (SF-36) & $40.54(10.31)$ & $43.76(12.19)$ & $\mathrm{T}(62)=2.66, \boldsymbol{p}=\boldsymbol{0 . 0 1 0}$ & 0.285 \\
MHC (SF-36) & $40.73(12.20)$ & $42.57(13.91)$ & $\mathrm{T}(62)=1.07, p=0.291$ & 0.151 \\
Dizziness-specific handicap (VHQ) & $48.70(19.76)$ & $39.49(24.46)$ & $\mathrm{T}(60)=3.60, \boldsymbol{p}=\boldsymbol{0 . 0 0 1}$ & 0.414 \\
VER (VSS-VER) & $0.93(0.50)$ & $0.77(0.60)$ & $\mathrm{T}(59)=2.07, \boldsymbol{p}=\mathbf{0 . 0 4 2}$ & 0.289 \\
Somatic AA (VSS-AA) & $1.31(0.74)$ & $1.31(0.89)$ & $\mathrm{T}(63)=0.03, p=0.974$ & 0.005 \\
\hline
\end{tabular}

ES indicates effect size. 


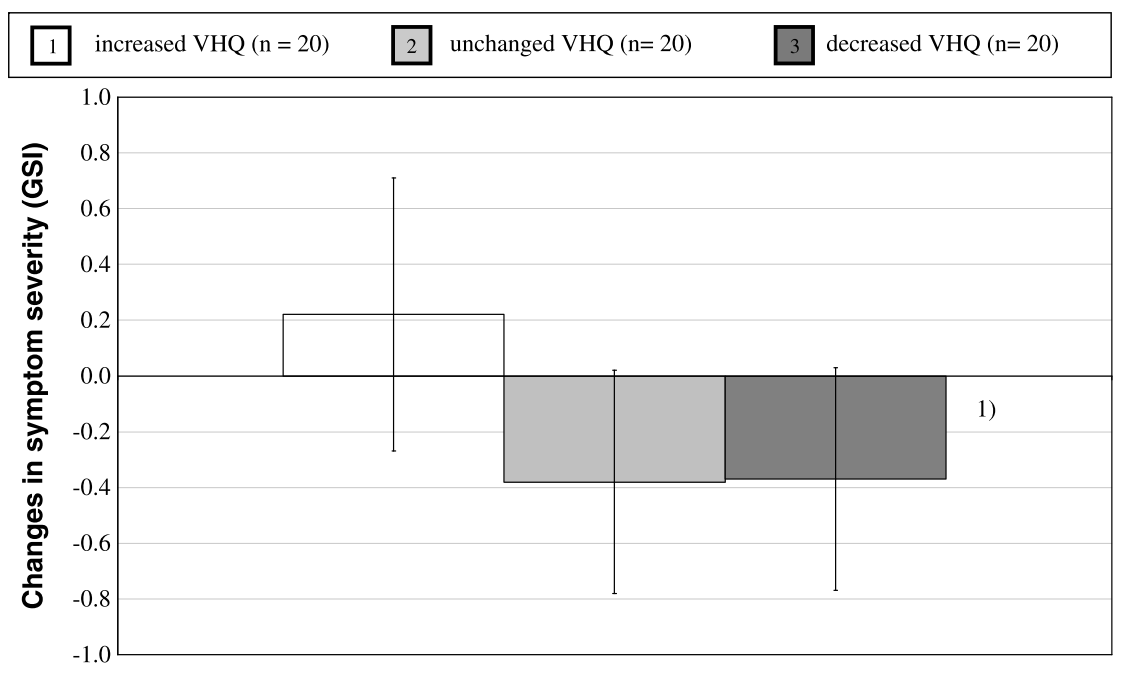

FIGURE 1. Changes in symptom severity (GSI) during the 3-year follow-up depending on changes in vertigo handicap (VHQ). 1) ANOVA: $F(2,59)=9.466, p<0.001 ;$ Scheffé's tests: $1>3$.

dizziness-specific handicap, and subjective QoL, and health care use, for example, additional diagnostic procedures and treatment modalities. To determine the prognostic features of the sample, we analyzed three severity subgroups of the VHQ with respect to dizziness and psychological strain. The aim of our study was to clarify the risk for chronicity and its influencing factors, on the one side, and to justify psychotherapeutic interventions, on the other side.

\section{Symptom Persistence}

Three years after intake examination, $36.9 \%(n=65)$ did not or only seldomly experienced ongoing dizziness or vertigo. A total of $63.1 \%$ developed chronic forms of primary SVD, for example, ongoing vertigo, imbalance, light-headedness, fainting, diffuse dizziness, or uncertainness. A comparison with literature was impossible because there are - to the best of our knowledge - no primary SVD reference groups in prospective follow-up studies. Former prospective studies investigating heterogeneous subgroups of vestibular vertigo syndromes, secondary SVD, specific phobic postural vertigo, or mixed etiological samples reported an improvement rate for vertigo and dizziness of between $70 \%$ and $80 \%$ (Huppert et al., 2005; Kroenke and Price, 1993; Yardley et al., 1998).

Our contrary findings must probably be explained by the current application of more detailed neuro-otological and psychosomatic procedures or by focusing on single diagnostic subgroups such as somatoform phobic postural vertigo (Huppert et al., 2005), which is often a secondary SVD. Unlike previous studies, we were able to reliably differentiate primary SVD and secondary SVD from vestibular vertigo syndromes. We therefore postulate that this difference in improvement rates reflects the specific characteristics of our primary (psychogenic) SVD sample. Vestibular vertigo syndromes or secondary SVD with a potentially better prognostic course of disease were omitted from our study. Nevertheless, Huppert et al. (2005) gave some evidence for our low recovery rate during 3 years. The authors reported full recovery in only $27 \%$ of phobic postural vertigo during the course of 8.5 years (Huppert et al., 2005). Kapfhammer et al. (1997) revealed $74 \%$ of psychological problems requiring specific psychiatric or psychotherapeutic interventions after a 2.5-year follow-up of phobic postural vertigo. Nazareth et al. (1999) examined the long-term progression of (mixed vestibular and psychogenic) dizziness in a general practice community study (Nazareth et al., 1999). After a period of 18 months, only $20 \%$ had improved. Our study therefore confirmed the high risk for chronicity even in a sample of primary SVD.

\section{Prevalence of Comorbid Mental Disorders}

Three years after admission, $40.7 \%$ of our patients with primary SVD had anxiety or phobic disorders, $21.5 \%$ were diagnosed

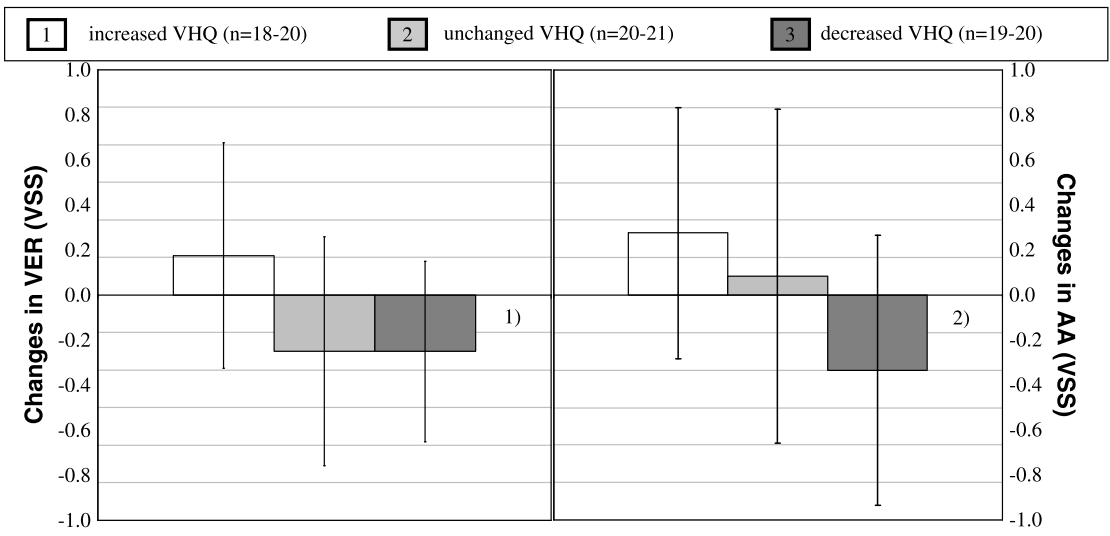

FIGURE 2. Changes in VER and AA (VSS) during the 3-year follow-up depending on changes in vertigo handicap (VHQ). 1) ANOVA: $F(2,56)=4.881, p=0.011$; Scheffé's tests: $1>2,3 ; 2)$ ANOVA: $F(2,60)=4.726, p=0.013$; Scheffé's tests: $1>3$. 


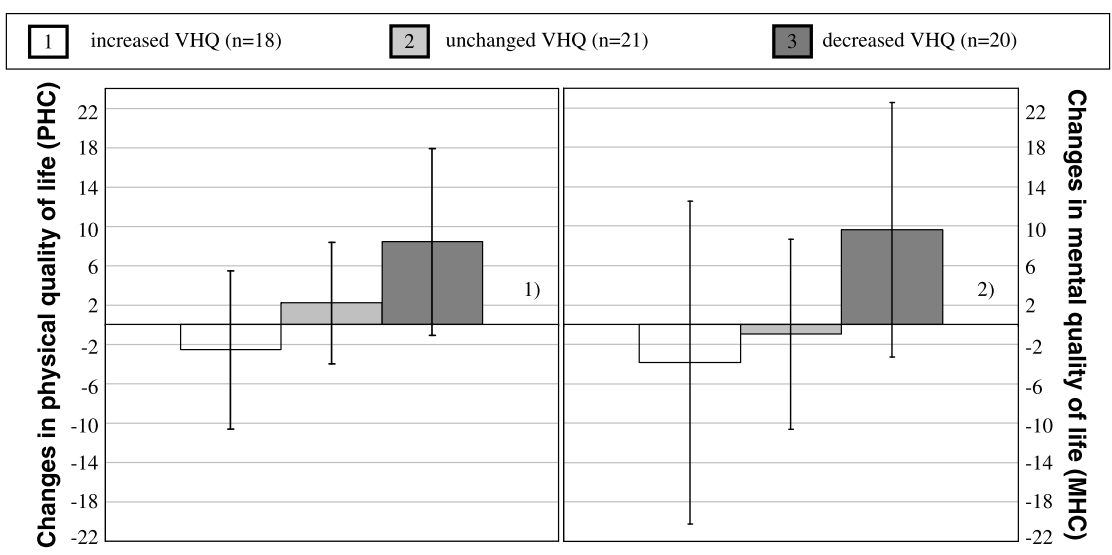

FIGURE 3. Changes in physical (PHC) and mental (MHC) QoL (SF-36) during the 3-year follow-up depending on changes in vertigo handicap (VHQ) 1) ANOVA: $F(2,58)=9.005, p<0.001$; Scheffé's tests: $3>1$; 2 ) ANOVA: $F(2,58)=5.753, p=0.005$; Scheffé's tests: $3>1,2$.

with depression, and $35.4 \%$ were diagnosed with somatoform disorders. Our analyses revealed no significant differences in all subgroups between the baseline and 3-year follow-up measurements. In correspondence with our data, previous studies postulated anxiety or phobic disorders as a main predictor for ongoing dizziness and vertigo (Asmundson et al., 1998; Wiltink et al., 2009; Yardley et al., 2001). Anxiety-related avoidance behavior significantly correlated with dizziness.

\section{Improvement of Symptom Severity and Physical Coping Versus Persistence of Autonomic Anxiety and Emotional Distress}

Regarding the course of primary SVD over time, the patients improved in dizziness-specific symptom severity (VSS-VER), handicap (VHQ), and physical QoL (PHC). However, the patients showed a change neither in general distress (GSI) nor in the mental QoL (MHC). Interestingly, the total group continued to experience high levels of autonomic anxiety and somatization tendency (VSS-AA). Staab and Ruckenstein (2007a) postulated the clinical syndrome of autonomic (exertional) dizziness as a frequent cause of chronic dizziness. Autonomic dysfunction including abnormal heart rate, blood pressure, or respiratory responses to stress factors even in the absence of syncopal or presyncopal symptoms were postulated as a subgroup of recurrent dizziness for lengthy periods of time (Staab and Ruckenstein, 2007a).

Our results lead us to the following questions: Which treatments did the patients with dizziness use during the period of 3 years? Why did they improve in physical parameters but not in mental comorbidity or distress?

\section{Treatment Modalities}

A total of $69.2 \%$ of the 65 patients with primary SVD received different forms of treatments: less than two thirds $(62.5 \%)$ received a psychotherapeutic one and more than half of the patients received a pharmacotherapeutic one (51.8\%). A large number of patients $(46.2 \%$ of $n=65$ ) searched redundant medical diagnostic procedures although they had been thoroughly informed about the reason and the course of their mental disorder. These patients underwent most frequently neurological $(40 \%)$, otolaryngological $(31.3 \%)$, and orthopedic $(28.1 \%)$ examinations. Despite the fact that the total sample received a recommendation for outpatient psychotherapy, less than half of the sample ( $45 \%$ of $n=65$ ) followed this recommendation for more than 3 months' duration. Summarizing these results, the patients with primary SVD ineffectively benefited from health care services but showed pronounced somatic illness representations leading to redundant medical contacts. With this in mind, chronicity was an unstoppable consequence of mistreatment. Exploring further prognostic features, we analyzed changes in handicap and coping with respect to psychopathology and QoL.

\section{Subgroup Analyses Derived From Different Handicap Developments}

The patients with no change or decrease in handicap significantly experienced lower levels of symptom severity (VSS-VER) and distress (GSI) compared with the patients with an increase in handicap. The patients with a decrease in handicap during a period of 3 years significantly improved in levels of autonomic anxiety (VSS-AA) and physical (PHC) and mental (MHC) health QoL. The patients who enhanced their coping capacity over time obtained more subjective well-being.

\section{Importance of the Data for Treatment}

On the basis of empirical evidence from our findings, we postulate disease-specific psychotherapy programs for patients with primary SVD. Diagnostic explanation and short psychoeducation in the present study did not suffice to reduce both the pronounced somatic illness representations and high health care use. Thus, the goals of prospective intervention programs are compliance, self-management, and empowerment, which are aspired not only by means of providing information and training skills but also by handling stress and life events, personal dimensions, somatic comorbidity, psychopathology, and doctor-patient relationship (Lahmann et al., 2012). In line with Yardley and Redfern (2001), we prefer a combination of psychotherapy with physiotherapy for dizziness. A common behavioral response to vertigo or dizziness is to avoid activities and situations that provoke symptoms. In addition, anxiety arousal and hyperventilation disinhibit somatic dizziness symptoms and trigger catastrophizing beliefs, focused body attention, and cognitive load. Yardley et al. (2001) therefore demonstrated the effectiveness of a combined vestibular rehabilitation therapy program focusing on negative beliefs of the consequences of dizziness. Andersson et al. (2006) successfully combined cognitive behavioral psychotherapy with vestibular rehabilitation in patients with chronic dizziness. Our working group showed that a combination of psychotherapeutic interventions with relaxation and vestibular rehabilitation significantly improved maladaptive illness perceptions. The 10 -session intervention for primary and secondary SVD led to both significantly higher feeling of personal control and lower levels of health care consumption in the 12-month follow-up (Tschan et al., 2012). Other treatment programs focused on the recovery of vestibular 
vertigo syndromes, phobic postural vertigo, or secondary SVD (Holmberg et al., 2006; Meli et al., 2007; Yardley et al., 2004). To the best of our knowledge, no international symptom- and complaintoriented psychotherapy program has been published for primary SVD.

\section{Limitations and Strengths of This Study}

Considering the dropout analyses, the dropout sample $(n=27)$ was significantly younger and more burdened by dizziness severity (VSS-VER) and distress (GSI) than was the 3-year follow-up sample. The main limitations address the relatively low sample size of 65 and the questionnaire survey without renewed clinical examinations at the 3 -year follow-up. Nevertheless, our response rate of $70.7 \%$ was in the range of studies with comparable follow-up durations (Bleichhardt et al., 2004; Linden et al., 2005; Wiltink et al., 2007). This is the first study exploring the long-term course of psychogenic dizziness in a relatively small but psychometrically and neuro-otologically wellcharacterized group of patients with primary SVD.

\section{CONCLUSIONS}

The findings of our study lead us to the following conclusions: a) Primary SVD poses a risk for chronicity; at the 3-year follow-up, almost two thirds still experienced ongoing dizziness. b) The patients with primary SVD showed pronounced somatic illness representations and used redundant diagnostic or medical procedures. c) The best long-term prognosis regarding symptom severity, dizzinessspecific anxiety, emotional distress, and QoL was found for the patients with a decreased handicap over time. We suggest the reduction of dizziness-specific handicap (VHQ) as an important factor for the long-term effectiveness of future psychotherapy programs.

\section{DISCLOSURES}

This work was supported by a grant of the Deutsche Forschungsgemeinschaft (EC 220/2-1).

The authors declare no conflict of interest.

\section{REFERENCES}

Andersson G, Asmundson GJ, Denev J, Nilsson J, Larsen HC (2006) A controlled trial of cognitive-behavior therapy combined with vestibular rehabilitation in the treatment of dizziness. Behav Res Ther. 44:1265-1273.

Asmundson GJ, Larsen DK, Stein MB (1998) Panic disorders and vestibular disturbance: An overview of empirical findings and clinical implications. J Psychosom Res. 44:107-120.

Best C, Eckhardt-Henn A, Tschan R, Dieterich M (2009) Psychiatric morbidity and comorbidity in different vestibular vertigo syndromes. Results of a prospective longitudinal study over one year. J Neurol. 256:58-65.

Bleichhardt G, Timmer B, Rief W (2004) Cognitive-behavioural therapy for patients with multiple somatoform symptoms - A randomised controlled trial in tertiary care. J Psychosom Res. 56:449-454.

Bullinger M, Kirchberger I (1998) Der SF-36 Fragebogen zum Gesundheitszustand: Handbuch für die deutschsprachige Fragebogenversion. Göttingen, Germany: Hogrefe.

Dieterich M, Brandt T (1993) Ocular torsion and tilt of subjective visual vertical are sensitive brainstem signs. Ann Neurol. 33:292-299.

Eckhardt-Henn A, Breuer P, Thomalske C, Hoffmann SO, Hopf HC (2003) Anxiety disorders and other psychiatric subgroups in patients complaining of dizziness. J Anxiety Disord. 17:369-388

Eckhardt-Henn A, Dieterich M (2005) Psychiatric disorders in otoneurologic patients. Neurol Clin. 23:731-749.

Franke G (1995) SCL-90-R: Die Symptom-Checkliste von Derogatis-Deutsche Version-Manual. Göttingen, Germany: Beltz.

Holmberg J, Karlberg M, Harlacher U, Rivano-Fischer M, Magnusson M (2006) Treatment of phobic postural vertigo. A controlled study of cognitive-behavioral therapy and self-controlled desensitization. Neurol. 253:500-506.

Honrubia V (1994) Quantitative vestibular function tests and the clinical examination. In Herdmann SJ (Ed), Vestibular rehabilitation (pp 113-164). Philadelphia: Davis.
Huppert D, Strupp M, Rettinger N, Hecht J, Brandt T (2005) Phobic postural vertigo-A long-term follow-up (5 to 15 years) of 106 patients. J Neurol. 252:564-569.

Kapfhammer HP, Mayer C, Hock U, Huppert D, Dieterich M, Brandt T (1997) Course of illness in phobic postural vertigo. Acta Neurol Scand. 95:23-28

Kroenke K, Hoffman RM, Einstadter D (2000) How common are various causes of dizziness? A critical review. South Med J. 93:160-167.

Kroenke K, Price RK (1993) Symptoms in the community: Prevalence, classification, and psychiatric comorbidity. Arch Intern Med. 153:2474-2480.

Lahmann C, Henningsen P, Dieterich M, Feuerecker R, Cyran CA, Schmid G (2012) The Munich diagnostic and predictor study of dizziness: Objectives, design, and methods. J Neurol. 259:702-711.

Linden M, Zubraegel D, Baer T, Franke U, Schlattmann P (2005) Efficacy of cognitive behaviour therapy in generalized anxiety disorders. Psychother Psychosom. $74: 36-42$.

Meli A, Zimatore G, Badaracco C, De Angelis E, Tufarelli D (2007) Effects of vestibular rehabilitation therapy on emotional aspects in chronic vestibular patients. $J$ Psychosom Res. 63:185-190.

Nazareth I, Landau S, Yardley L, Luxon L (2006) Pattern of presentation of dizziness in primary care-A cross-sectional cluster analyses study. J Psychosom Res. 60:395-401.

Nazareth I, Yardley L, Owen N, Luxon L (1999) Outcome of symptoms of dizziness in a general practice community sample. Fam Pract. 16:616-618.

Neuhauser HK, Radtke A, von Brevern M, Lezius F, Feldmann M, Lempert T (2008) Burden of dizziness and vertigo in the community. Arch Intern Med. $168: 2118-2124$.

Neuhauser HK, von Brevern M, Radtke A, Lezius F, Feldmann M, Ziese T, Lempert T (2005) Epidemiology of vestibular vertigo: A neurotologic survey of the general population. Neurology. 65:898-904.

Staab JP, Ruckenstein MJ (2007a) Autonomic nervous system function in chronic dizziness. Otol Neurotol. 28:854-859.

Staab JP, Ruckenstein MJ (2007b) Expanding the differential diagnosis of chronic dizziness. Arch Otolaryngol Head Neck Surg. 133:170-176.

Tschan R, Eckhardt-Henn A, Scheurich V, Best C, Dieterich M, Beutel ME (2012) Steadfast-Effectiveness of a cognitive-behavioral self-management program for patients with somatoform vertigo and dizziness. Psychother Psychosom Med Psychol. 62:111-119.

Tschan R, Wiltink J, Best C, Bense S, Dieterich M, Beutel ME, Eckhardt-Henn A (2008) Validation of the German version of the Vertigo Symptom Scale (VSS) in patients with organic or somatoform dizziness and healthy controls. J Neurol. 255:1168-1175.

Tschan R, Wiltink J, Best C, Beutel ME, Dieterich M, Eckhardt-Henn A (2010) Validation of the German version of the Vertigo Handicap Questionnaire (VHQ) in patients with vestibular vertigo syndromes or somatoform dizziness. Psychother Psychosom Med Psychol 60:385-390.

Wiltink J, Dippel A, Szczepanski M, Thiede R, Alt C, Beutel ME (2007) Long term weight loss maintenance after inpatient psychotherapy of severe obesity based on a randomized study - Predictors and maintaining factors of health behaviour. J Psychosom Res. 62:691-698.

Wiltink J, Tschan R, Michal M, Subic-Wrana C, Eckhardt-Henn A, Dieterich M, Beutel ME (2009) Dizziness: Anxiety, health care utilization and health behaviour-Results from a representative German community survey. $J$ Psychosom Res. 66:417-424.

Wittchen HU, Burke JD, Semler G, Pfister H, Von Cranach M, Zaudig M (1989) Recall and dating of psychiatric symptoms. Test-retest reliability of timerelated symptom questions in a standardized psychiatric interview. Arch Gen Psychiatry. 46:437-443.

Yardley L (2000) Overview of psychologic effects of chronic dizziness and balance disorders. Otolaryngol Clin North Am. 33:603-616.

Yardley L, Beech S, Weinman J (2001) Influence of beliefs about the consequences of dizziness, and the effect of therapy on beliefs. J Psychosom Res. 50:1-6.

Yardley L, Donovan-Hall M, Smith HE, Walsh BM, Mullee M, Bronstein AM (2004) Effectiveness of primary care-based vestibular rehabilitation for chronic dizziness. Ann Intern Med. 141:598-605.

Yardley L, Owen N, Nazareth I, Luxon L (1998) Prevalence and presentation of dizziness in a general practice community sample of working age people. $\mathrm{Br} \mathrm{J} \mathrm{Gen}$ Pract. 48:1131-1135.

Yardley L, Redfern MS (2001) Psychological factors influencing recovery from balance disorders. J Anxiety Disord. 15:107-119. 